\title{
Proficient Energy Efficient A2S Leach Technique in Wireless Sensor Networks
}

\author{
Saravanakumar. R, Susila. S. G, Krishnachandrika. S
}

\begin{abstract}
In Wireless Sensor Networks (WSNs) lifetime of the system relies upon the vitality of the hubs, where vitality utilization is for the most part utilized for information transmission as opposed to detecting and preparing. A critical test in remote sensor systems is the ideal utilization of hub assets. Bunching of sensor hubs helps to utilize the hub vitality ideally and delay the lifetime of vitality compelled remote sensor arrange. Also, in WSN, the decision of steering convention assumes a significant job in using the vitality of hubs effectively. In this paper, another A2S LEACH (Active $\leftrightarrow$ Sleep - Low Energy Adaptive Clustering Hierarchy) directing strategy is proposed, which joins the two significant classes of various leveled conventions in particular bunch based methodology and chain based methodology. The proposed system is reproduced utilizing NS2 and the outcomes are examined. Reenactment results shows that the proposed A2S LEACH steering convention altogether diminishes vitality utilization and expands the all-out lifetime of the remote sensor organize when contrasted with the LEACH convention.

Keywords: Wireless Sensor Networks, LEACH, A2S LEACH, Node Scheduling, NS2
\end{abstract}

\section{INTRODUCTION}

Remote sensor systems have been distinguished as one of the most noticeable advancements for the present period. Microelectronics specialized advances have made the sensor arranges progressively appropriate for those territories where traditional systems fizzle or are insufficient.

WSN are ordinarily conveyed in the zones like military applications, science and industry applications, for example, savvy transportation, social insurance, climate checking, calamity recuperation, natural life observing, security systems, modern and building computerization and space investigation.

Normally, a WSN comprises of huge number of sensor hubs. A sensor hub comprises of four significant subsystems 1) Sensing subsystem, for social event the information from the earth,

2) Processing and memory subsystem, for information preparing and information putting away, all sensors have constrained memory and restricted handling speed,

3) Wireless correspondence subsystem, for information transmission and gathering,

4) Energy supply subsystem, which is the non-batterypowered force hotspot for the sensor hub.

Revised Manuscript Received on May 27, 2020.

* Correspondence Author

Saravanakumar.R*, Professor, Dept.of ECE, Vivekanandha College of Engineering for Women, Namakkal Dt., 637205.

Susila.S.G, Assistant Professor, Dept.of ECE, University College of Engineering BIT Campus, Tirucirappalli. 620 024. susinila@gmail.com

Krishnachandrika.S, PG scholar, Power System Engineering, Vivekanandha College of Engineering for Women, Namakkal Dt., 637205. rsksusi@gmail.com

(C) The Authors. Published by Blue Eyes Intelligence Engineering and Sciences Publication (BEIESP). This is an open access article under the CC BY-NC-ND license (http://creativecommons.org/licenses/by-nc-nd/4.0/)
Among these, the vitality supply subsystem assumes a significant job in the lifetime of the system, in light of the fact that the sensor hubs consume the greater part of their vitality on transmitting the information as opposed to preparing and sensing [1]. So the steering procedure running on the WSN ought to devour the assets proficiently. The usually proposed directing methods are as of now disseminated among different classes which are level, various leveled and area based systems. In each class a few vitality effective arrangements were put out. The utilization of a progressive structure is one methodology that is probably going to succeed. Intricate and significant issues, for example, vitality effective help, channel conflict, dormancy and the executives of WSNs should be routed to empower huge scope organizations. Numerous vitality productive arrangements have been put out in each class. The utilization of a various leveled framework is one methodology that would succeed. Mind boggling and significant issues, for example, vitality productive help, channel dispute, idleness and the board of WSNs should be handled to empower huge scope organizations. Various leveled association of enormous sensor systems is the premise for many techniques that addresses these issues.

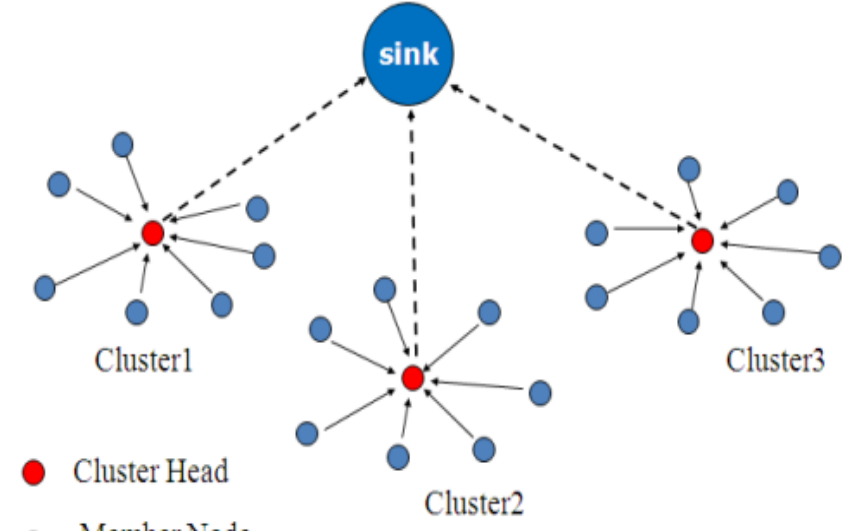

Member Node

Fig.1.1 Network model with clustering

Various leveled association of sensor arrange as appeared in Fig.1.1, prompts three various types of steering conventions dependent on how they conveyed to the base station, they are Clustering approach, Chain based methodology and Tree based Approach. Essential contender for bunch based setup is applications that spread expansive sensor fields and empower information collection. The system is disintegrated into a progression of groups in bunch based arrangement; each group contains arrangement of hubs just as a bunch head for bunch the board. In a few bunching based methodologies, the hubs inside a group just interface in their $\mathrm{CH}$. The $\mathrm{CHs}$ are answerable for sorting out coordination between the groups and the intra-bunches.

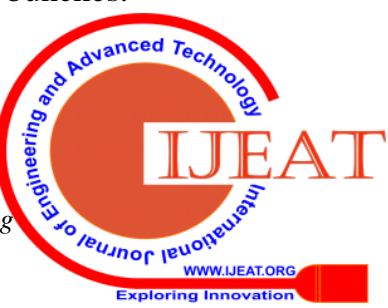


Contact between $\mathrm{CHs}$ can be through single jumps or different bounces. Drain, LEACH-C, V-LEACH, HEED, CBRP and the TEEN protocols [2] are models for bunch based setups. Albeit many bunching arrangements have been proposed, just a couple of consolidates grouping with an information directing plan that is proper for enormous scope WSNs [3]. The bunch based arrangement issues are picked for each round, new cluster head $(\mathrm{CH})$, which expands overhead group creation. In some bunching conventions cluster head legitimately speak with base station, which lead to vitality wastage.

The system can be isolated into little districts dependent on the areas in chain based approaches. A single data forwarding chain will be created for forwarding the data to the base station or multiple small chains are constructed for efficient based methodologies. A solitary information sending chain will be made for sending the information to the base station or various little chains are built for effective tasks. PEGASIS [9] and CHIRON conventions are the models for chain based routing [4]. The issue with the chain-based methodology is the making of long chain for information transmission that prompts bottle neck correspondence and early demise of sensor hubs close to base stations. The proposed calculation composes the system into groups dependent on hubs leftover vitality, likelihood and the normal lingering vitality of all nodes [5]. To beat the grouping overhead issue, the proposed calculation keeps the equivalent $\mathrm{CHs}$ for a few rounds. On the off chance that the CHs lingering vitality falls underneath the limit esteem, at that point the new CHs are chosen. To spread the whole bunch with least number of hubs, this paper presents another rest plan for group hubs, so that vitality can be spared altogether. Cluster heads (CHs) gathers the information from the sensor utilizing TDMA and totals the information. At least two chains are developed among the $\mathrm{CHs}$ for information transmission to the base station.

\section{GENERAL APPROACHES OF ENERGY CONSERVATION}

Until the elevated level grouping of vitality preservation proposition is tended to, it merits introducing the system and hub level design which apply from a sensor organize viewpoint, which is the most broadly acknowledged model in the writing.

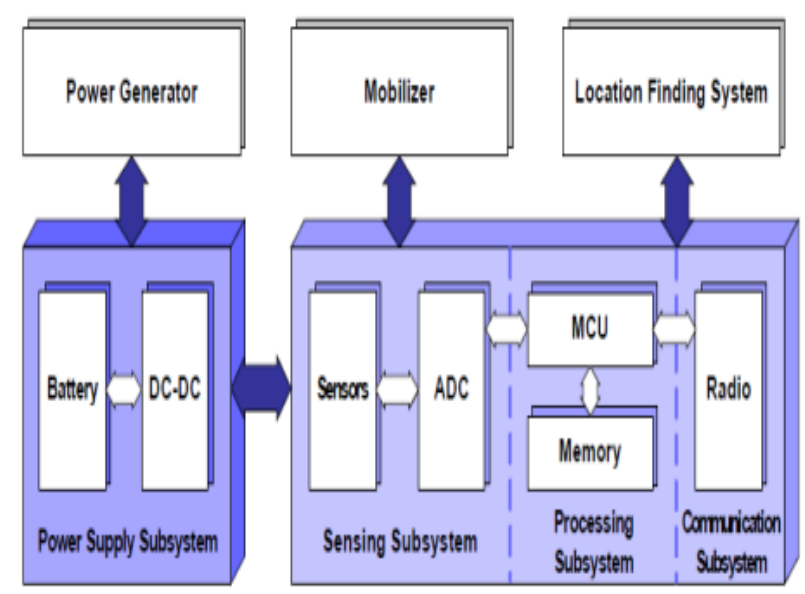

Fig.2.1 Wireless sensor node architecture
On the other side, Fig.2.1 shows the engineering of a run of the mill remote sensor hub, as generally expected in the literature[6].Depending on the particular application, sensor hubs may likewise incorporate extra segments, for example, allocation discovering framework to decide their position, a mobilizer to change their area or arrangement (e.g., antenna's direction, etc. In any case, as the last segments are discretionary, and just every so often utilized, it won't consider in the accompanying conversation. Clearly, the force separate vigorously relies upon the particular hub. This uncovers a Mote-class hub's capacity qualities are totally not the same as those of a star entryway hub. In any case, the accompanying comments for the most part hold. The correspondence subsystem has vitality utilization far higher than the processing subsystem. It has been indicated that transmitting the slightest bit may expend as much as executing a thousand directions. Subsequently, correspondence ought to be exchanged for calculation.

The radio vitality utilization is of a similar request in the gathering, transmission and inert states, while the force utilization drops of at least one request for size in the sleep state. The radio ought to either be taken care of (or killed) at whatever point conceivable. The detecting gadget might be another huge wellspring of vitality utilization relying upon the specific application, so its capacity utilization likewise should be decreased. In light of the previously mentioned plan and force breakdown, numerous techniques must be used to lessen power utilization in remote sensor systems, even simultaneously. The characterized three key empowering procedures at a general level, in particular duty cycling, information driven methodologies and versatility.

Assignment cycling is basically focused towards the systems administration subsystem. The most productive vitality monitoring activity is to situate the radio handset in rest mode (low-power) if no correspondence is required. In a perfect world, the radio ought to be turned off when information is done being sent/got, and ought to be continued when another information bundle is accessible. Contingent upon organize activity hubs switch among dynamic and rest cycles along these lines. Regularly, this movement is called obligation cycling, and the obligation cycle is described as the portion of time hubs that are dynamic all through their lifetime. Since the sensor hubs play out a joint capacity, their rest/wake-up times must be facilitated.

Snoozing/wakeup booking calculation in this way goes with any obligation cycling plan. As a rule, it is an appropriated calculation dependent on which sensor hubs decide when to change from dynamic to rest and in reverse. It makes the enactment of neighboring hubs simultaneously, permitting the exchange of bundles practical in any event, when hubs work with a low obligation cycle (for example they rest a significant part of the time).

Obligation cycling plans are normally unconscious of information being gotten to by means of sensor hubs. Therefore, information driven techniques can be utilized to additionally help the vitality effectiveness. In fact, information detecting impacts on sensor hubs' vitality utilization in two different ways:

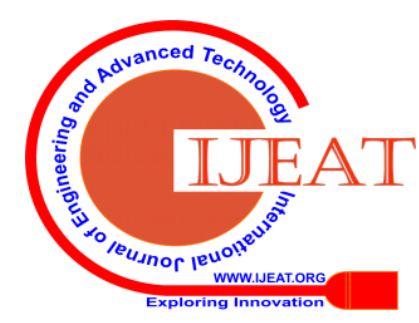


Un required examples: There is typically a decent spatial and additionally fleeting association between tested information, so there is no compelling reason to transmit the repetitive data to the sink.

Power utilization of the detecting subsystem: This isn't sufficient to decrease contact when the sensor itself is power hungry.

Unneeded examples bring about unnecessary vitality utilization in the main occasion, despite the fact that the examining costs are irrelevant in light of the fact that they add to unneeded correspondences.

The subsequent issue happens when there is no negligible utilization of the detecting subsystem ever. Information guided strategies talked about beneath are intended to limit the measure of information gathered by keeping up the exactness of the detecting inside a suitable range for application. If a portion of the sensor hubs are versatile, portability can at last be utilized as a vitality utilization moderation strategy (past obligation cycling and information had driven procedures). Bundles originating from sensor hubs in a static sensor arrange follow a multi-bounce way toward the sink(s). Accordingly, a couple of ways can be stacked more than others and hubs closer to the sink need to transfer more bundles with the goal that they are progressively defenseless to consumption of untimely vitality (channeling impact). In the event that any of the hubs (counting, most likely, the sink) are portable, the traffic stream might be adjusted if cell phones are explicitly answerable for gathering information from static nodes. Ordinary hubs anticipate the cell phone's entry and course messages to it, so the correspondences happen in closeness (legitimately or if nothing else with an insignificant multijump navigate). Subsequently, conventional hubs can spare assets, as way length, dispute and overhead sending are additionally diminished. Be that as it may, because of interchanges, the cell phone will visit the system in order to disseminate vitality utilization all the more consistently. On the off chance that the expense of assembling sensor hubs is restrictive, the ordinary arrangement is to associate sensor hubs to people, for example, transports or creatures, which will wander the detecting region in anyway.

\section{LITERATURE SURVEY}

Jiguo Yua.,et al.,2012.[7] a cluster-based routing protocol for non-uniform node distributed wireless sensor networks is proposed, which includes an energy-aware clustering algorithm EADC and a cluster-based routing algorithm.

Gholam Hossein Ekbatanifard.,et al.,2012 [8]focuses on adaptive quorum-based MAC protocol, Queen-MAC. This protocol separately and adaptively schedules nodes wakeuptimes, decreases idle listening and collisions, increases network throughput, and extends network lifetime.

Ungjin Jang.,et al., 2012[9] discusses the problem of arranging the sleep-wake cycles of nodes in a tree collecting data under time limit constraints.

An optimal wake-up frequency assignment (OWFA) algorithm that takes into account the data rate at each node and the total permissible delay, after officially modeling the problem being addressed.

J.Zhang.,et al., [10] described the Effective data aggregation supported by dynamic routing in wireless sensor networks, IEEE International Conference on Communications May 2010, is proposed a clustering-based routing protocol that minimizes global energy use by distributing the load at different points in time to all nodes.

A fixed clustering based approach to prolong the lifetime of sensor networks employing data aggregation is described in I.F.Akyildiz, W.Su, Y.Sankarasubramaniam, E.Cayirci, [11] Wireless sensor networks: a survey, Elsevier Science Journal of Computer Networks, 2002.

C.Jaikaeo and C.Chien-Chung Shen, [12] Sensor Information Networking, International Journal of Wireless networks, Wiley Publication, 2005 RDAG is an approach of Data aggregation by reducing number of transmissions which are an effective energy-saving method by using the LEACH definition.

Kyounghwa Lee et al.,[13] presented A Density and Distance based Cluster Head Selection Algorithm in Sensor Networks, 2010 ICACT2010 is proposed Energy Efficient Algorithm for Cluster-Head Selection is basically a modification of LEACH algorithm.

While there are numerous algorithms that came up saying LEACH adjustment, it is supposed to be more energy efficient and more uniformly distribute energy charge [14]. Growing node in the network determines whether or not to become a cluster head for the current round based on the percentage of cluster heads suggested for the network and how many times the node has been a cluster head up to now. The node must pick a random number between zero and one, the node becomes a cluster-head for the current round if the number is less than a threshold. Cluster formation is based on residual energy and cost. Our proposed method supports sleep and active scheduling to reduce the energy consumption.

Energy consumption on demand helps in the efficient usage of the network as well as increasing the lifespan of the network by reducing energy use. None of the previous research has, to the best of our knowledge, incorporated all these considerations in one single work.

\section{PROPOSED PROTOCOL}

\subsection{A2s Leach Routing Protocol}

In this paper, an A2S LEACH bunching calculation dependent on likelihood and remaining vitality of the hub is proposed which use the vitality of the hub proficiently.

Grouping is an instrument for productive information trade and for vitality proficiency in remote sensor systems (WSNs). Bunching implies gathering sensor hubs and choosing one hub as a Cluster head $(\mathrm{CH})$, so group hubs transmit their detected information to the CHs. CHs gather the information, order it and forward amassed information for additional consideration to the preparing focus called base station.

Bunching gives better asset use and limits vitality utilization in WSNs by diminishing the quantity of sensor hubs that partake in significant distance transmission [15].

The working of the proposed approach is given in equation (1). For Simulation, the accompanying presumptions are made

There are $\mathrm{M}$ hubs in the sensor field.

Number of clusters is $\mathrm{K}$.

In each cluster average number of sensor nodes is $\mathrm{N}$

Where $\mathrm{N}=\mathrm{M} / \mathrm{K}$.

Blue Eyes Intelligence Engineering \& Sciences Publication

(C) Copyright: All rights reserved. 
That of the nodes after $\mathrm{N}$ rounds must once have been a cluster head $(\mathrm{CH})$. A unique $\mathrm{Ni}$ identifier is assigned to every node for all $\mathrm{i}=0,1,2,3,4$...M-1.

\subsection{Algorithm for Cluster Heads Selection}

Initially, all hubs are the same,i.e. there are no $\mathrm{CHs}$ in each bunch, $\mathrm{j}=0$ where the counter $\mathrm{j}$ is $\mathrm{CH}$. A qi hub is browsed all hubs and the accompanying advances are constantly executed: First, q expands I by 1 and check whether I is even, that hub is chosen as the $\mathrm{CH}$ for that round and declares its new residence to all part hubs in the bunch; in any case, on the off chance that I is odd, it can't be a CH for that round and it will hold up until the following adjust and be prepared to acknowledge the new $\mathrm{CH}$ commercial message.

For that cycle, a fixed worth (edge esteem) is set for the new $\mathrm{CH}$ to transmit. At the point when the worth surpasses the edge, $\mathrm{j}$ is increased by 1 and the choice procedure for new $\mathrm{CH}$ starts. It tests if the accompanying two conditions hold.

1 . For past (1/p)-1 rounds a sensor node has not been a cluster head.

2. The residual energy of a node is more than the average energy of all the sensor nodes in the clustering. Thus, the selection value (SV) of a node becoming new cluster head is given in equation (2).

$$
S V(i)=\frac{p \operatorname{Erem}(i) * K}{1-p * r \bmod \frac{1}{p} \operatorname{Eavg}(i) * N}
$$

Where,

$P$ is the likelihood that the node will become the head of the cluster,

$r$ is the current round number,

$E_{\text {rem }}$ is the remaining energy in the node $i E_{\text {avg }}$ is the total energy of all the nodes in a cluster. It carries on until $\mathrm{j}=\mathrm{K}$, If $\mathrm{j}=\mathrm{K}$ the algorithm ends.

The node with the highest selection value is the new head of the cluster. The latest $\mathrm{CH}$ gathers aggregates and transmits the sensed data from member nodes and the compressed data to the next cluster head or base station.

\subsection{Cluster Formation}

After CHs have been picked, the subsequent stage in the grouping procedure is bunch framing. Underneath gives the depiction of new group development.

Step1: The new cluster heads communicates a minimal effort control messages to all non-group hubs in the system utilizing Carrier Sense Multiple Access/Collision Detection (CSMA/CD) MAC Protocol.

Step2: Which sensor hub chooses which groups it will join dependent on the RSS (Receiver Signal Strength) by picking $\mathrm{CH}$ which needs least correspondence vitality?

Step3: Every non-group hub utilizes CSMA/CD to communicate something specific back to the $\mathrm{CHs}$ educating them regarding the bunch they wish to have a place with.

Step4: The bunch head fixes the limit subsequent to getting the messages from all hubs, and lingerie the $\mathrm{CH}$ data to its individuals.

Ordinarily, CHs are picked on each round in pretty much every bunching calculation, this prompts overhead grouping on each round. To beat this proposed approach keeps the equivalent $\mathrm{CHs}$ for a few rounds. In the event that the $\mathrm{CHs}$ remaining vitality falls underneath the limit esteem, at that point the new $\mathrm{CHs}$ are picked. For reproduction, $0.1 \mathrm{~J}$ is considered as edge esteem, ie on the off chance that the $\mathrm{CH}$ has $0.4 \mathrm{~J}$ at first, and in the event that hub arrives at $0.3 \mathrm{~J}$, at that point the grouping methodology is again instated.4.4. Sleep Schedule for Cluster Nodes.

In WSN, rest plan was applied to hub sensors to spare the hub's assets. A few hubs are in dynamic state in a bunch, and a few hubs are in rest mode. The significant issue is to cover the whole bunch with least number of sensors.

It's exceptionally hard to ensure full inclusion for a checked region, regardless of whether all sensors are on-dynamic. Inclusion instrument is to pick a subset of dynamic hubs to hold the presumption of inclusion.

This makes another inclusion in the bunch, which chooses some dynamic hubs in groups while holding the group's inclusion suspicion.

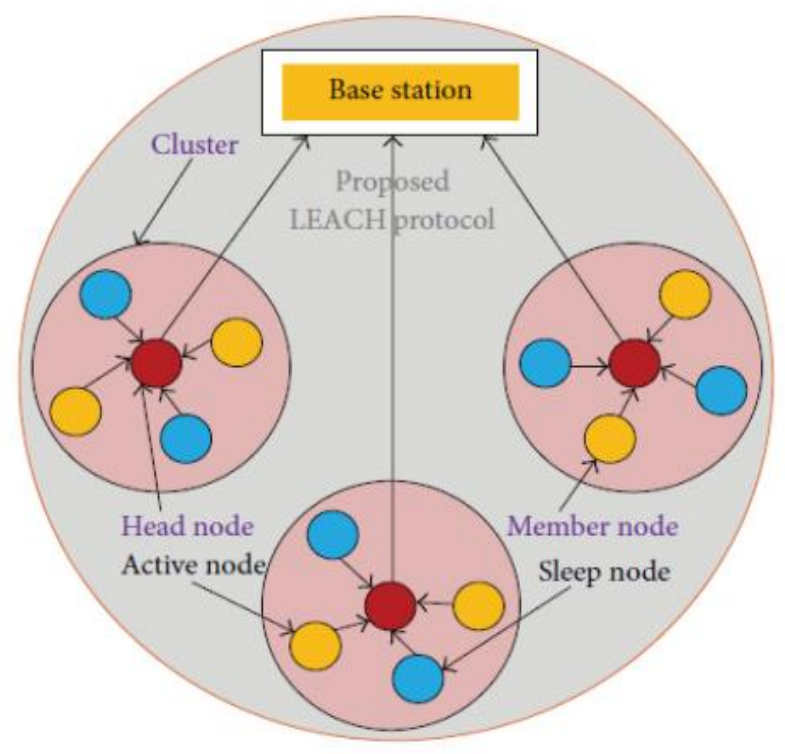

Fig.4.1 Node scheduling Technique

In Fig.4.1 shows hub booking method, here the hubs are requested based by their vitality's and the separation between the hub and the cluster head.

Choose and change the hubs which have the least assets. The dynamic hubs possess the whole system and other part hubs are killed, bringing about extensively diminished vitality utilization in intra-group hubs, and expanded system life. The related vacancy outline is appeared in Fig.4.2. Here, each round of recreation in the consistent state odd number hubs is dynamic and considerably number hubs are idle that implies in rest state. Next round of reenactment odd number hubs are dormant and much number hubs are dynamic.

\subsection{Information sending chains development}

Stage 1: Cluster heads send their position data by means of Route Request message to base station after group arrangement.

Stage 2: As chain individuals, the base station chooses a few $\mathrm{CHs}$ dependent on position data (typically $\mathrm{CHs}$ closer to base station). Then again, as in PEGASIS, it utilizes the avaricious calculation to render the little strings.

Stage 3: Give the chains to the CHs by base station.

Step4: All cluster heads send their information down the chain to the pioneer hub, in the end the pioneer hub moves the information got to the base station.

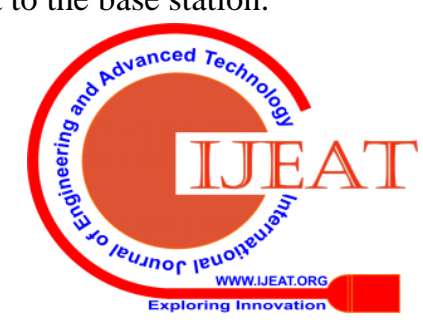




\subsection{Information Collection and Data Forwarding}

Step1: The $\mathrm{CH}$ gets ready Time Division Multiple Access (TDMA) planning table and send it to all hubs in the group. This message contains time assigned to every hub to transmit to the $\mathrm{CH}$ with in each group.

Step2: Each sensor hub utilizes TDMA distributed to it to transmit information to the $\mathrm{CH}$ with a solitary bounce transmission and switch off its handset at whatever point the separation between the hub and $\mathrm{CH}$ is something other than one vitality sparing hop.

Step3: CHs will give new TDMA spaces to all hubs in their bunches when dispensed time for $\mathrm{G}+\mathrm{R}$ has passed, for every hub to know specific time it will transmit information during transmission to forestall information impact, which may expand vitality utilization. $G$ is the absolute time required for the $\mathrm{CH}$ to gather the information from its all bunch individuals and $\mathrm{R}$ is the arbitrary time interim, during that time the CHs handset is killed.

Step4: The $\mathrm{CH}$ performs information combination work by dispensing with repetitive information after the sum total of what information has been acquired, and packs the information into a solitary parcel.

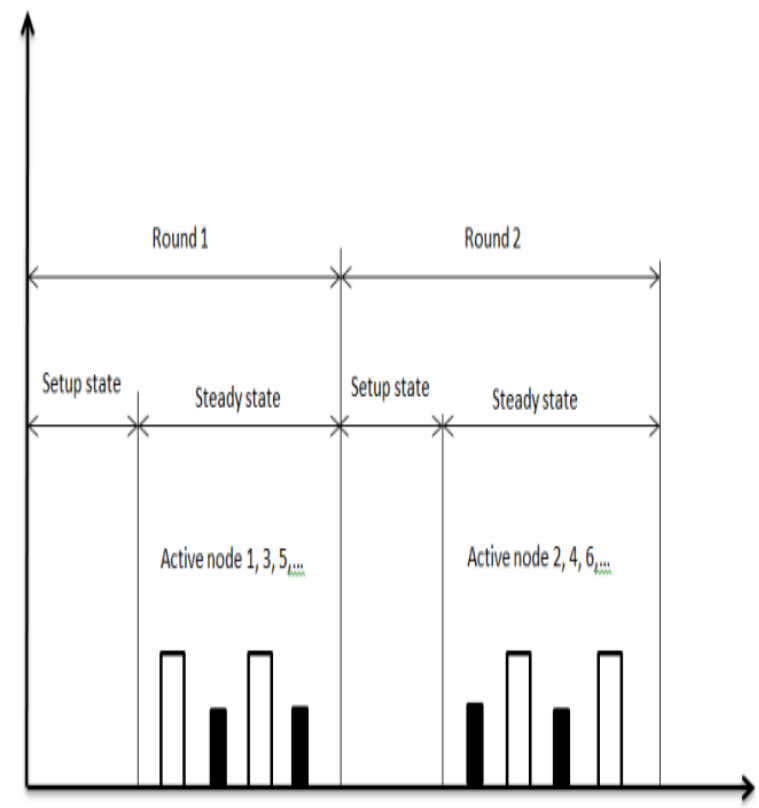

Fig.4.2 Time Slot Diagram

\section{SIMULATION RESULT}

The used simulation parameters are placed in Table 5.1.

\subsection{Simulation Parameters}

Table 5.1

\begin{tabular}{|l|l|}
\hline Parameter & Amplitute \\
\hline Simulation time & 7000 Seconds \\
\hline Duty cycle & $5 \%, 25 \%, 40 \%$ \\
\hline Node idle power & $100 \mathrm{~mW}$ \\
\hline Node Rx power & $100 \mathrm{~mW}$ \\
\hline Node Tx power & $100 \mathrm{~mW}$ \\
\hline Node sleep power & $1 \mathrm{~mW}$ \\
\hline Transition power & $20 \mathrm{~mW}$ \\
\hline Transition time & $5 \mathrm{~ms}$ \\
\hline Energy model & $\mathrm{NS} 2$ \\
\hline
\end{tabular}

5.2 Energy Level Comparison Table
Table 5.2

\begin{tabular}{|c|c|c|}
\hline Protocol & Time(ms) & $\begin{array}{c}\text { Remaining Network } \\
\text { Energy(J) }\end{array}$ \\
\hline LEACH & 50 & 35 \\
\hline A2S LEACH & 50 & 45 \\
\hline
\end{tabular}

\subsection{Values of Performance Metrics}

Table 5.3

\begin{tabular}{|c|c|c|c|c|c|c|}
\hline $\begin{array}{r}\text { Time(m } \\
\text { () }\end{array}$ & Thro & hput & $\begin{array}{r}\mathbf{P a} \\
\mathbf{D e} \\
\mathbf{R}\end{array}$ & $\begin{array}{l}\text { ket } \\
\text { ery } \\
\text { io } \\
\end{array}$ & & $\begin{array}{l}\text { End } \\
\text { ay }\end{array}$ \\
\hline Protoco & $\underset{\text { CH }}{\text { LEA }}$ & $\begin{array}{c}\text { A2S } \\
\text { LEA } \\
\text { CH }\end{array}$ & $\begin{array}{c}\text { LEA } \\
\mathrm{CH}\end{array}$ & $\begin{array}{c}\text { A2S } \\
\text { LEA } \\
\text { CH }\end{array}$ & $\begin{array}{l}\text { LEA } \\
\mathrm{CH}\end{array}$ & $\begin{array}{c}\text { A2S } \\
\text { LEA } \\
\text { CH }\end{array}$ \\
\hline 10 & 56 & 62 & 0.2 & 0.16 & 0.9 & 0.7 \\
\hline 20 & 102 & 116 & 0.4 & 0.42 & 15 & 16.7 \\
\hline 30 & 152 & 167 & 0.6 & 0.67 & 21 & 25.7 \\
\hline 40 & 170 & 186 & 0.8 & 0.93 & 58 & 43.4 \\
\hline 50 & 248 & 259 & 1.0 & 1.48 & 98 & 87.3 \\
\hline
\end{tabular}

\subsection{Message Broadcasting}

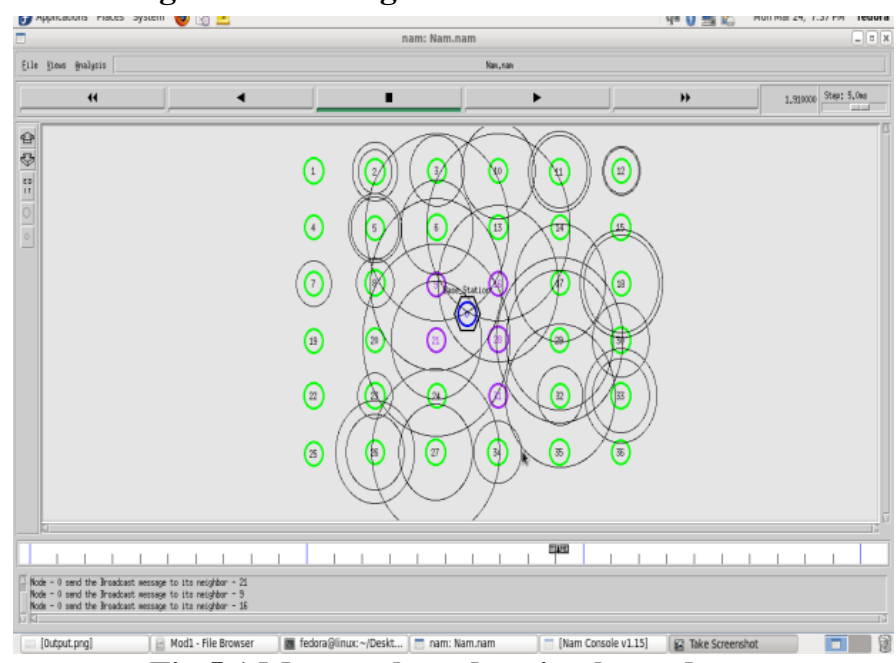

Fig.5.1 Message broadcasting by nodes

After 50ms of simulation time in LEACH and A2S

$\mathrm{LEACH}$, the remaining network energy level is high in A2S LEACH, depicted in Table 5.2. The Table 5.3 is evidenced the throughput, packet delivery ratio and end to end delay after simulation. The A2S LEACH is having good values than LEACH protocol in all the three aspects. The Fig.5.1 illustrates message broadcasting by sensor nodes. Here node scheduling (active/sleep) system is incorporated in Fig.5.2. The better energy consumption of A2S LEACH in simulation is illustrated in graph as shown in Fig.5.3. Similarly the better throughput is depicted in graph as shown in Fig.5.4. 


\subsection{Node Scheduling}

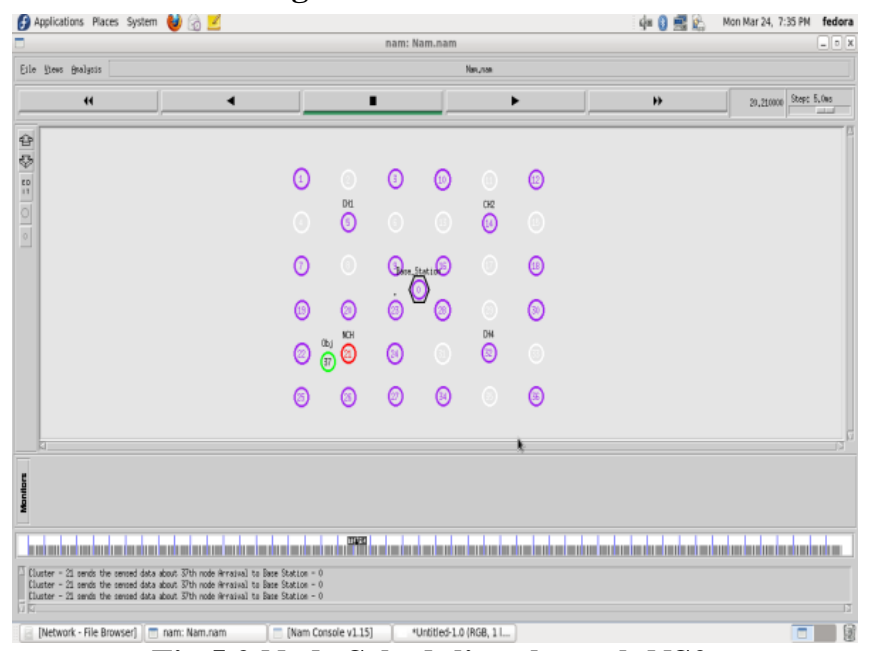

Fig.5.2 Node Scheduling through NS2

\subsection{Energy Consumption versus Time}

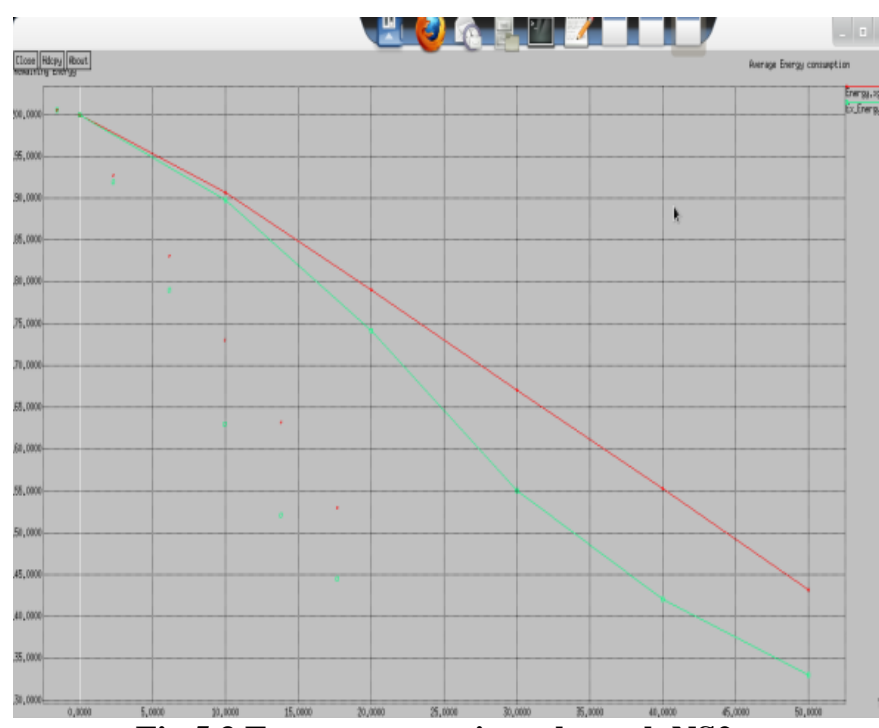

\subsection{Throughput versus Time}

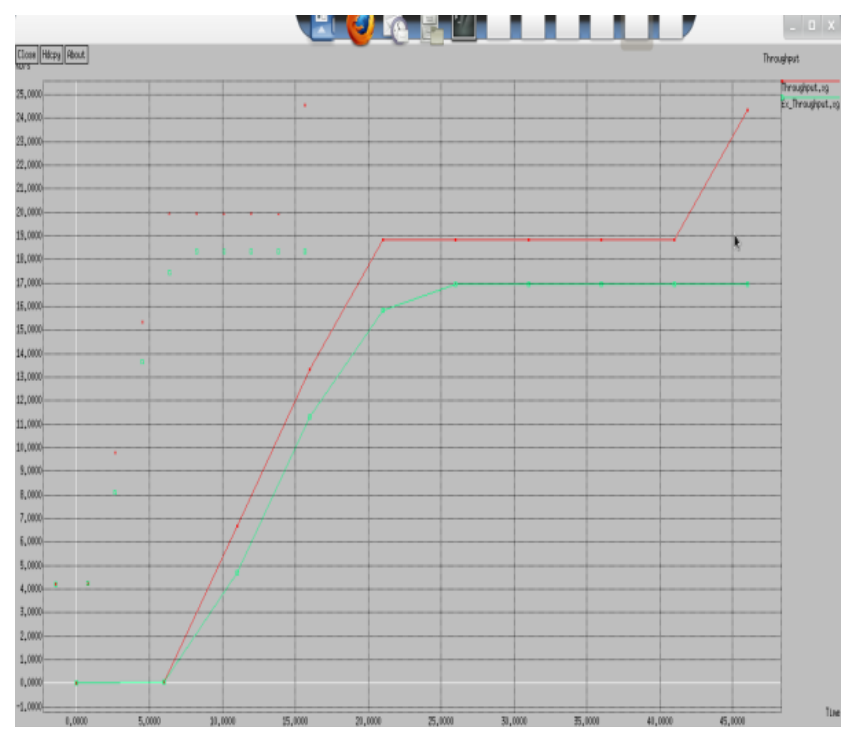

Fig.5.4 Throughput

\section{CONCLUSION}

In this paper, the proposed A2S LEACH protocol using the proper node scheduling (ACTIVE and SLEEP) in individual cluster of the whole network, which is an energy efficient clustering method for WSNs and compared it with the normal LEACH protocol. Results from our simulations using NS2 are shows that the proposed protocol provides better performance in energy efficiency and increasing level in lifetime of the wireless sensor networks.

\section{REFERENCES}

1. W.Heinzelman, A.Sinha, A.Wang and A.Chandrakasan, "EnergyScalable Algorithms and Protocols for Wireless Microsensor Networks, "International Conference on Acoustics, Speech and Signal Processing ICASSP, vol.6, Istanbul, Turkey, 2000, pp.3722-3725.

2. A.Manjeshwar and D.P.Agrawal, TEEN: a routing protocol for enhanced efficiency in wireless sensor networks, in Proceedings of the $15^{\text {th }}$ International Parallel and Distributed Processing Symposium, pp.2009-2015, SanFrancisco, Calif, USA, April2001.

3. K.Akkaya, F.Senel and B.McLaughlan, Clustering of wireless sensor and actor networks based on sensor distribution and connectivity, Journal of Parallel and Distributed Computing, vol.69, no.6, pp.573587, 2009.

4. S.Lindesy and C.Raghavendra, "PEGASIS: Power-Efficient Gathering in Sensor Information System, "in Proceedings of the Aerospace Conference, IEEE, vol.3, BigSky, Montana, 2002,pp.9-16.

5. YongchangYu and YichangSong, An Energy-Efficient Chain-Based Routing Protocol in Wireless Sensor Network, 2010 International Conference on Computer Application and System Modeling (ICCASM2010), Taiyuan, 978-1-4244-7237-6/C2010, IEEE, pp.V11486-V11-489.

6. T.Shu and M.Krunz, Coverage-time optimization for clustered wireless sensor networks: a power-balancing approach, IEEE/ACM Transactions on Networking, vol.18, no.1, pp.202-215, 2010.

7. W.B.Heinzelman, A.P.Chandrakasan and H.Balak-rishnan, An application-Specific Protocol Architecture for Wireless Microsensor Networks, IEEE Transactions on Wireless Communications, Vol.1, No.4, 2002, pp.660-670. doi:10.1109/TWC.2002.804190.

8. Jiguo Yua, Yingying Qi, Guanghui Wang, Xin Gua, “A cluster-based routing protocol for wireless sensor networks with non-uniform node distribution", International Journal of Electronics and Communications (AEU), Vol. 66, Issue. 1, January 2012, pp. 54-61.

9. Gholamhossein Ekbatanifard, R. Monsefi, Mohammah-H Yaghmaee and Seyed Amin Hosseini Seno, "Queen-MAC: A quorum-based energy-efficient medium access control protocol for wireless sensor networks" Computer Networks, 56(8):2221-2236 - May2012, DOI: 10.1016/j.comnet.2012.03.004.

10. Jang, Ungjin, Sunggu Lee, and Sungjoo Yoo, "Optimal wake-up scheduling of data gathering trees for wireless sensor networks", Journal of Parallel and Distributed Computing, pp.536-546, 2012.

11. J. Zhang, Q. Wu, F. Ren, T. He, and C. Lin., "Effective data aggregation supported by dynamic routing in wireless sensor networks", IEEE International Conference on Communications (ICC'10), May 2010, pp 55-64.

12. I.F. Akyildiz, W. Su, Y. Sankarasubramaniam, E. Cayirci, "Wireless sensor networks: a survey", Elsevier Science Journal of Computer Networks, 2002, pp 393-422.

13. C.Jaikaeo and C.Chien-Chung Shen, "Sensor Information Networking", International Journal of Wireless networks, Wiley Publication, Vol 12, Issue 3, 2005, pp 22- 47.

14. Kyounghwa Lee, Joohyun Lee, Hyeopgeon Lee, Yongtae Shin, "A Density and Distance based Cluster Head Selection Algorithm in Sensor Networks, Feb.7-10, 2010 ICACT2010 pp.162-165.

15. W.R.Heinzelman, A.Chandrakasan and H.Balakrishnan, EnergyEfficient Communication Protocol for Wireless Microsensor Networks, Proc. of the Hawaii International Conference on System Science, Jan.4,2000.

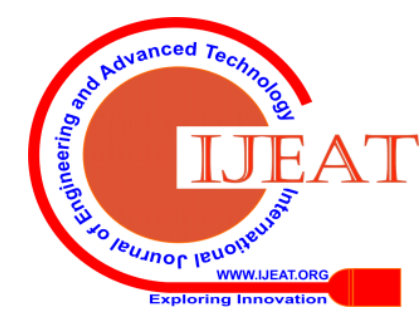




\section{AUTHORS PROFILE}

Saravanakumar.R working as Professor in Electronics and Communication Engineering department at Vivekanandha College of Engineering for Women, Tiruchengode, Tamilnadu for the past four years to till date. He completed his BE (ECE) in 1995 and ME (Communication Systems) in 2001 from Bharathidasan University and acquired his Ph.D from Anna University Chennai in 2014. He has nearly 25 years of engineering college teaching experience. He has published 24 papers in national and international conferences and 12 papers in international journals. He is a recognized research supervisor in Anna University in Faculty of Information and Communication. He guided nearly 60 UG and PG level projects so for.

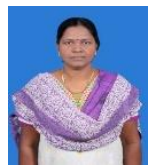

Susila.S.G working as Visiting faculty in Electronics and Communication Engineering department at University College of Engineering BIT campus Tiruchirappalli, Tamilnadu, India for the past ten years to till date. She completed her BE (ECE) in 1996 and ME (Communication Systems) in 2014 from Bharathidasan University and acquired her Ph.D from Anna University Chennai in 2019. She has nearly 15 years of engineering college teaching experience. He has published 12 papers in national and international conferences and 08 papers in international journals. He guided nearly 49 UG and PG level projects so for.

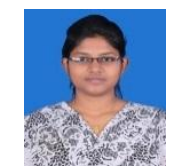

Krishnachandrika.S pursuing ME (Power System Engineering) in Vivekanandha College of Engineering for Women, Tiruchengode, Tamilnadu. She completed her BE (EEE) in 2019 Anna University Chennai. She took three inconferences. plant training and presented two papers in international 